\title{
The All-or-Nothing Nature of Two-Party Secure Computation
}

\author{
Amos Beimel $^{1}$, Tal Malkin ${ }^{2,3}$, and Silvio Micali ${ }^{2}$ \\ 1 Division of Engineering and Applied Sciences \\ Harvard University, 40 Oxford st., Cambridge, MA 02138 \\ beimel@deas.harvard .edu
}

Supported by grants ONR-N00014-96-1-0550 and ARO-DAAL03-92-G0115.

2 Laboratory for Computer Science, Massachusetts Institute of Technology 545 Technology sq., Cambridge, MA 02139

3 tal@theory.lcs.mit.edu

Supported by DARPA grant DABT63-96-C-0018.

\begin{abstract}
A function $f$ is computationally securely computable if two computationally-bounded parties Alice, having a secret input $x$, and Bob, having a secret input $y$, can talk back and forth so that (even if one of them is malicious) (1) Bob learns essentially only $f(x, y)$ while (2) Alice learns essentially nothing.

We prove that, if any non-trivial function can be so computed, then so can every function. Consequently, the complexity assumptions sufficient and/or required for computationally securely computing $f$ are the same for every non-trivial function $f$.
\end{abstract}

\section{Introduction}

Secure Computation. Let $f$ be a two-argument finite function, that is, $f$ : $S_{1} \times S_{2} \rightarrow S_{3}$ (where $S_{1}, S_{2}$, and $S_{3}$ are finite sets), and let Alice and Bob be two possibly malicious parties, the first having a secret input $x \in S_{1}$ and the second having a secret input $y \in S_{2}$. Intuitively, securely computing $f$ means that Alice and Bob keep turns exchanging message strings so that (1) Bob learns the value $z=f(x, y)$, but nothing about $x$ (which is not already implied by $z$ and $y$ ), no matter how he cheats, while (2) Alice learns nothing about $y$ (and thus nothing about $z$ not already implied by $x$ ), no matter how she cheats.

In a sense, therefore, a secure computation of $f$ has two constraints: a correctness constraint, requiring that Bob learns the correct value of $f(x, y)$, and a privacy constraint, requiring that neither party learns more than he/she should about the other's input.

Throughout this paper, any function to be securely computed is a finite, two-argument function.

The One-Sidedness of Secure Computation. The notion of secure computation informally recalled above is the traditional one used in the two-party, malicious model (cf., GMW87 Section 4.2], and Kil88 Kil90). This notion is 
"one-sided" in that only Bob learns the result of computing $f$, while Alice learns nothing. Such one-sidedness is unavoidable in our malicious model. In principle, one could conceive of a more general notion of secure computation in which "both Alice and Bob learn $f(x, y)$ ㄹ " However, such a more general notion is not achievable in a two-party, malicious model: the first party who gets the desired result, if malicious, may stop executing the prescribed protocol, thus preventing the other from learning $f(x, y)^{\mathbf{2}}$ Moreover, such a malicious party can terminate prematurely the execution of the prescribed protocol exactly when he/she "does not like" the result.

Trivial and Non-Trivial Functions. A function $f$ is called trivial if it can be securely computed even if a cheating party has unlimited computational power, and non-trivial otherwise.

An example of a trivial function is the "projection of the first input"; namely the function $\mathrm{P}_{1}:\{0,1\} \times\{0,1\} \rightarrow\{0,1\}$ so defined: $\mathrm{P}_{1}\left(b_{0}, b_{1}\right)=b_{0}$. Another example is the "exclusive-or function"; namely, the function XOR : $\{0,1\} \times$ $\{0,1\} \rightarrow\{0,1\}$ so defined: $\operatorname{XOR}\left(b_{0}, b_{1}\right)=b_{0}+b_{1} \bmod 2$. Indeed, a secure way of computing either function consists of having Alice send her secret bit to Bob. This elementary protocol clearly is a correct and private way of computing $\mathrm{P}_{1}$. It also is a correct and private way of computing XOR. Indeed, Alice's revealing her own secret bit $b_{0}$ enables Bob to compute locally and correctly the desired XOR of $b_{0}$ and $b_{1}$. Moreover, Alice's revealing $b_{0}$ also satisfies the privacy constraint: Bob could deduce Alice's bit anyway from the output of the XOR function he is required to learn.

An example of a non-trivial function is the function AND : $\{0,1\} \times\{0,1\} \rightarrow$ $\{0,1\}$ so defined: $\operatorname{AND}\left(b_{0}, b_{1}\right)=b_{0} \wedge b_{1}$. Another non-trivial function is the (chosen 1-out-of-2) oblivious transfer; namely, the function OT : $\{0,1\}^{2} \times\{0,1\} \rightarrow$ $\{0,1\}$ so defined: $\mathrm{OT}\left(\left(b_{0}, b_{1}\right), i\right)=b_{i}$, that is, Bob only learns the bit of Alice he chooses. (The non-triviality of these functions follows from CK89.)

Secure Computability of Non-Trivial Functions. By definition, securely computing non-trivial functions is conceivable only when (at least one of) Alice and Bob are computationally bounded, but by no means guaranteed. Nonetheless, a series of results have established that secure computation of non-trivial functions is possible under complexity assumptions of various strengths. In particular,

- The OT function is securely computable under the assumption that integer factorization is computationally hard Rah81 FMR84 FGL 8.5 Cré88 3

${ }^{1}$ Or even a more general scenario where Bob learns $f(x, y)$ while Alice learns $g(x, y)$.

${ }^{2}$ Or $g(x, y)$ in the more general scenario.

${ }^{3}$ Rabin Rab81 introduced a variant of the oblivious transfer, the random oblivious transfer, and provided an implementation of it which is provably secure in the honest-but-curious model. Fischer, Micali, and Rackoff F'MIR84 improved his protocol so as to be provably secure against malicious parties. Even, Goldreich, and 
- All functions are securely computable if factoring is hard Yao86; and, actually,

- All functions are securely computable if any trapdoor permutation exists GMW 4

Such results raise fundamental questions about the strength of the computational assumptions required for secure computation. In particular,

Q1: What assumption is required for securely computing at least one non-trivial function?

Q2: What assumption is required for securely computing a given non-trivial function $f$ ?

Q3: Are there assumptions sufficient for securely computing some non-trivial function $f$ but not sufficient for securely computing some other non-trivial function $g$ ?

Completeness for Secure Computation. Another important result is that the OT function is complete for secure computation Kil88 By this we mean that, if OT is securely computable, then so are all functions. A bit more specifically, given any function $f$ and any protocol securely computing OT, one can efficiently and uniformly construct a protocol securely computing $f$.

The completeness of the OT function raises additional fundamental questions. In particular,

Q4: Are there other (natural) functions that are complete for secure computation? Q5: Is there a (natural) characterization of the functions complete for secure computation?

\subsection{Main Results}

A Characterization of Complete Functions. In this paper we prove the following

Main Theorem: Any non-trivial function is complete for secure computation.

Lempel EGL85 introduced the notion of the chosen 1-out-of-2 oblivious transfer, together with an implementation of it which is provably secure in the honest-butcurious model. Finally, Crépeau Crés8 showed how to transform any secure protocol for the random oblivious transfer to a secure protocol for the chosen 1-out-of- 2 oblivious transfer.

4 The hardness of factoring implies the existence of trapdoor permutations, but the vice-versa might not hold.

${ }^{5}$ Kilian Kil91 also proves a more general result, but in a different model, which we discuss in Subsection IL.2 
Clearly, our result provides an explicit and positive answer to questions $Q 4$ and $Q 5$, and an explicit and negative answer to $Q 3$. Our result also provides an implicit answer to questions $Q 1$ and $Q 2$. Namely, letting $f$ be any given non-trivial function, and $A_{f}$ be the assumption that $f$ is securely computable:

For any non-trivial function $g$, assumption $A_{f}$ is both necessary and sufficient for securely computing $g$.

An Interpretation of Our Main Theorem. Our main theorem also suggests that just assuming the existence of one-way functions may be insufficient to guarantee secure computation. Let us explain. Impagliazzo and Rudich IR89 show that, without also proving that $\mathcal{P} \neq \mathcal{N} \mathcal{P}$, no protocol having oracle-access to a random function can be proved to compute the OT function securely. This result has been interpreted as providing strong evidence that "one-way functions are not sufficient for constructing a protocol securely computing the OT function." It is then according to the same interpretation that our main theorem suggests that, for any non-trivial function $f, A_{f}$ should be stronger than the existence of one-way functions.

A Characterization of Trivial Functions. Is there a combinatorial property that makes a two-argument function securely computable by two, possibly malicious, parties with unbounded computing power? In our paper we also provide such a characterization (actually crucial to the proof of our main theorem 6 ) in terms of insecure minors.

We say that $f$ contains an insecure minor if there exist inputs $x_{0}, y_{0}, x_{1}, y_{1}$ such that $f\left(x_{0}, y_{0}\right)=f\left(x_{1}, y_{0}\right)$ and $f\left(x_{0}, y_{1}\right) \neq f\left(x_{1}, y_{1}\right)$, and prove:

Main Lemma: A two-argument function $f$ is trivial if and only if $f$ does not contain an insecure minor.

\subsection{Comparison to Previous Work}

The Honest-But-Curious Model. Both completeness and characterization of non-trivial functions have been extensively investigated with respect to a weaker notion of two-party secure computation introduced in GMW87: the honest-but-curious mode 1 In this model, the parties are guaranteed to properly execute a prescribed protocol, but, at the end of it, each of them can use his/her own view of the execution to infer all he/she can about the other's input. In this model, because no protocol can be prematurely terminated, it is meaningful to consider "two-sided" secure computation of a function $f$; that is, one in which each party learns $f(x, y)$, but nothing else about the other's input that is not

\footnotetext{
${ }^{6}$ Note that our main theorem provides a characterization of both trivial and nontrivial functions, though not a combinatorial-looking one!

${ }^{7}$ Originally called "the semi-honest model" in GMW 87
} 
already implicit in $f(x, y)$ and his/her own input. Indeed this is the traditional notion of secure function computation in the honest-but-curious model.

Similar to the malicious model, a function is said to be trivial in the honestbut-curious model if it can be securely computed even if the two (honest-butcurious) parties have unbounded computing power, and non-trivial otherwise. The above mentioned results of Yao86 GMW87 immediately imply that every two-argument function is securely computable in the honest-but-curious model, under the corresponding complexity assumptions (hardness of factoring and existence of trapdoor permutations).

A combinatorial characterization of the trivial functions in the honest-butcurious model was first given by Chor and Kushilevitz CK89 for Boolean functions (i.e., predicates), and then by Kushilevitz Kus89 for all functions.

While in the malicious model we prove that all non-trivial functions are complete, in the honest-but-curious one the "corresponding" theorem does not hold; there exists a (non-Boolean) function that is neither trivial nor complete Kus89 Kil91 KKMO98 On the other hand, Kilian, Kushilevitz, Micali, and Ostrovsky KKMO98 prove that any non-trivial Boolean function is complete in the honest-but-curious model.

Kilian's MODEL. In Kil91 Kilian characterizes the functions $f$ that are complete in an hybrid model of secure computation. Namely, the functions $f$ for which, given access to a two-sided black-box for $f$ (i.e., one giving the result $f(x, y)$ to both Alice and Bob), one can construct, for any function, a onesided protocol that is information-theoretically secure against unbounded malicious parties. He proves that these functions $f$ are exactly those containing an embedded-or, a special case of our insecure minor (i.e., one satisfying the additional constraint $\left.f\left(x_{0}, y_{0}\right)=f\left(x_{0}, y_{1}\right)\right)$.

In sum, Kilian's result "reduces" standard (one-sided) protocols to two-sided black boxes. Notice that this is different (and not applicable) to our case, where we reduce standard protocols to standard protocols. (Indeed, our characterization of the complete function is different, and there are functions that are complete in our setting but not in his.)

Also notice that two-sided black boxes might be implementable via "tamperproof hardware" or in some other physical model, but, as explained above, no protocol can securely implement a two-sided black box for a function $f$ against malicious parties $\overline{9}$

$\overline{8}$ KKMO98 prove this by combining the following two results. Kus89 shows an example of a function which is non-trivial yet does not contain an embedded or, and Kil91 shows that a function that does not contain an embedded or cannot be complete in this model. We note that this example is a function which contains an insecure minor, and thus is complete in the malicious (one-sided) model, as we prove in this paper.

9 Two-sided boxes may instead be implemented by protocols (under certain complexity assumptions) in the honest-but-curious model. 
Reduction Models. Black-box reductions (as those of CK88 Kil91 KKMO98) are an elegant way to build new secure protocols. While two-sided boxes are not implementable by secure protocols against malicious parties, one-sided black boxes can be (under certain complexity assumptions). Thus, one may consider completeness under one-sided black box reductions. However, as we shall point out in Section 4.2 such reductions are not strong enough to solve the questions we are interested in. We thus use an alternative definition of a reduction that is natural for protocols secure against bounded malicious parties. Informally, for us a reduction is a transformation of a given secure protocol for $f$ (rather than a one-sided black box for $f$ ) into a protocol for $g$ secure against computationally bounded malicious parties.

Organization. In Section $\boldsymbol{Z}$ we define protocols, and secure computation in the unbounded honest-but-curious model. In Section 3 we provide a definition of secure computation in the unbounded malicious model, and proceed to characterize the trivial functions. Finally, in Section 4 we characterize the complete functions, and prove that any non-trivial function is complete.

\section{Preliminaries}

\section{$2.1 \quad$ Protocols}

Following GMR85, we consider a two-party protocol as a pair, $(A, B)$, of Interactive Turing Machines (ITMs for short). Briefly, on input $(x, y)$, where $x$ is a private input for $A$ and $y$ a private input for $B$, and random input $\left(r_{A}, r_{B}\right)$, where $r_{A}$ is a private random tape for $A$ and $r_{B}$ a private random tape for $B$, protocol $(A, B)$ computes in a sequence of rounds, alternating between $A$-rounds and $B$-rounds. In an $A$-round ( $B$-round) only $A$ (only $B$ ) is active and sends a message (i.e., a string) that will become an available input to $B$ (to $A$ ) in the next $B$-round ( $A$-round). A computation of $(A, B)$ ends in a $B$-round in which $B$ sends the empty message and computes a private output 10

Transcripts, Views, And Outputs. Letting $E$ be an execution of protocol $(A, B)$ on input $(x, y)$ and random input $\left(r_{A}, r_{B}\right)$, we define:

- The transcript of $E$ consists of the sequence of messages exchanged by $A$ and $B$, and denoted by $\operatorname{TRANS}^{A, B}\left(x, r_{A}, y, r_{B}\right)$

- The view of $A$ consists of the triplet $\left(x, r_{A}, t\right)$, where $t$ is $E$ 's transcript, and denoted by $\operatorname{VIEW}_{A}^{A, B}\left(x, r_{A}, y, r_{B}\right)$;

- The view of $B$ consists of the triplet $\left(y, r_{B}, t\right)$, where $t$ is $E$ 's transcript, and denoted by $\operatorname{VIEW}_{B}^{A, B}\left(x, r_{A}, y, r_{B}\right)$;

- The output of $E$ consists of the string $z$ output by $B$ in the last round of $E$, and denoted by $\mathrm{OUT}_{B}\left(y, r_{B}, t\right)$, where $t$ is $E$ 's transcript.

${ }_{10}$ Due to the one-sidedness of secure computation, only machine $B$ produces an output. 
In all the above the superscript $(A, B)$ will sometimes be omitted when clear from the context.

We consider the random variables $\operatorname{TRANS}\left(x, \cdot, y, r_{B}\right), \operatorname{TRANS}\left(x, r_{A}, y, \cdot\right)$ and $\operatorname{TRANS}(x, \cdot, y, \cdot)$, respectively obtained by randomly selecting $r_{A}, r_{B}$, or both, and then outputting $\operatorname{TRANS}\left(x, r_{A}, y, r_{B}\right)$. We also consider the similarly defined random variables $\operatorname{VIEW}_{A}\left(x, \cdot, y, r_{B}\right), \quad \operatorname{VIEW}_{A}\left(x, r_{A}, y, \cdot\right)$, $\operatorname{VIEW}_{A}(x, \cdot, y, \cdot), \operatorname{VIEW}_{B}\left(x, \cdot, y, r_{B}\right), \operatorname{VIEW}_{B}\left(x, r_{A}, y, \cdot\right)$, and $\operatorname{VIEW}_{B}(x, \cdot, y, \cdot)$,

\subsection{Secure Computation in the Unbounded Honest-but-Curious Model}

Among all notions of secure computation, the one for two unbounded honestbut-curious parties is the simplest one to formalize. In this model the parties Alice and Bob are guaranteed to follow the prescribed protocol $(A, B)$ (namely they use the right ITMs $A$ and $B$ ), but may try to obtain as much information as they can from their own views. Intuitively, a protocol is secure in this model if the following conditions hold: (1) Bob learns the value $z=f(x, y)$, but nothing about $x$ (not already implied by $z$ and $y$ ), while (2) Alice learns nothing about $y$ (and thus nothing about $z$ not already implied by $x$ ). A formal definition follows.

Definition 1. Let $f: S_{1} \times S_{2} \rightarrow S_{3}$ be a finite function. A protocol $(A, B)$ securely computes $f$ against unbounded honest-but-curious parties, if the following conditions hold:

1. Correctness: $\forall x \in S_{1}, \forall y \in S_{2}, \forall r_{A}, \forall r_{B}$, letting $v=\operatorname{VIEW}_{B}^{A, B}\left(x, r_{A}, y, r_{B}\right)$,

2. Privacy:

$$
\mathrm{OUT}_{B}(v)=f(x, y) \text {. }
$$

Alice's Privacy: $\forall x_{0}, x_{1} \in S_{1}, \forall y \in S_{2}, \forall r_{B}$, if $f\left(x_{0}, y\right)=f\left(x_{1}, y\right)$ then

$$
\operatorname{VIEW}_{B}^{A, B}\left(x_{0}, \cdot, y, r_{B}\right)=\operatorname{VIEW}_{B}^{A, B}\left(x_{1}, \cdot, y, r_{B}\right) \text { 11 }
$$

Bob's Privacy: $\forall x \in S_{1}, \forall y_{0}, y_{1} \in S_{2}, \forall r_{A}$,

$$
\operatorname{VIEW}_{A}^{A, B}\left(x, r_{A}, y_{0}, \cdot\right)=\operatorname{VIEW}_{A}^{A, B}\left(x, r_{A}, y_{1}, \cdot\right) .
$$

\section{A Combinatorial Characterization of Trivial Functions}

So far, we have intuitively defined a trivial function to be one that is computable by a protocol that is secure against unbounded malicious parties ${ }^{12}$ Combinatorially characterizing trivial functions, however, requires first a quite formal notion of secure computation in our setting, a task not previously tackled. This is what we do below.

\footnotetext{
${ }^{11}$ Equivalently, the corresponding transcripts are identically distributed (and similarly below).

12 By this we do not mean that the parties participating in a protocol computing a trivial function are computationally-unbounded, but that the "privacy and correctness" of their computation holds even when one of them is allowed to be malicious and computationally-unbounded.
} 


\subsection{Secure Computation in the Unbounded Malicious Model}

In this model Alice or Bob may be malicious, namely cheat in an arbitrary way, not using the intended ITM $A$ (or $B$ ), but rather an arbitrary (computationally unbounded) strategy $A^{\prime}$ (or $B^{\prime}$ ) of their choice. The definition of secure computation in the malicious model requires some care. For example, it is not clear how to define what the input of a malicious party is.

We handle the definition of secure computation in the spirit of MR92 (a definition primarily aimed at secure computation in a multi-party scenario, such as $\mathrm{BGW} 88 \mathrm{CCD88}$ ). Intuitively, we require that when Alice and Bob are honest then Bob computes the function $f$ correctly relative to his own input and Alice's input. We also require that when Bob is honest and for any possible malicious behavior of Alice, Bob computes the function $f$ correctly relative to his own input and Alice's input as defined by evaluating a predetermined input function on Alice's view of the joint computation. Because the computation is one-sided and a malicious Bob might not output any value, the correctness requirement is limited to the above two cases. Finally, we require privacy for an honest Alice against a possibly malicious Bob, and privacy for an honest Bob against a possibly malicious Alice.

Definition 2. Let $f: S_{1} \times S_{2} \rightarrow S_{3}$ be a finite function. A protocol $(A, B)$ securely computes $f$ against unbounded malicious parties, if the following conditions hold:

1. Correctness: $\forall x \in S_{1}, \forall y \in S_{2}, \forall r_{A}, \forall r_{B}$,

Correctness when both Alice and Bob are honest:

Letting $v=\operatorname{VIEW}_{B}^{A, B}\left(x, r_{A}, y, r_{B}\right)$, then $\operatorname{OUT}_{B}(v)=f(x, y)$.

Correctness when only Bob is honest: For every strategy $A^{\prime}$ there is $\mathcal{I}_{A^{\prime}}:\{0,1\}^{*} \rightarrow S_{1}$ such that, letting

$$
\begin{gathered}
v_{A^{\prime}}^{\prime}=\mathrm{VIEW}_{A^{\prime}}^{A^{\prime}, B}\left(x, r_{A}, y, r_{B}\right) \text { and } v_{B}^{\prime}=\operatorname{VIEW}_{B}^{A^{\prime}, B}\left(x, r_{A}, y, r_{B}\right), \\
\operatorname{OUT}_{B}\left(v_{B}^{\prime}\right)=f\left(\mathcal{I}_{A^{\prime}}\left(v_{A^{\prime}}^{\prime}\right), y\right)
\end{gathered}
$$

2. Privacy:

Alice's Privacy: For every strategy $B^{\prime}, \forall x_{0}, x_{1} \in S_{1}, \forall y \in S_{2}, \forall r_{B}$, if

$$
f\left(x_{0}, y\right)=f\left(x_{1}, y\right)
$$

$\overline{13}$ By the previous condition, the mapping $\mathcal{I}_{A}$ (i.e., for honest Alice) gives a "correct" input, which is either $x$ itself or "equivalent" to $x$, in the sense that it yields the same output $f(x, y)$. Notice that for secure computation of a function $f$ we may restrict the protocol so that Bob always ouputs a value that is compatable with his input. That is, on input $y$ Bob outputs a value $z$ such that there is some $x$ for which $f(x, y)=z$ (indeed, Bob before outputing $z$ can always check for compatibility and output $f(0, y)$ otherwise). When restricted to these secure computation the correctness for honest Bob and Alice implies the correctness when only Bob is honest, that is, the function $\mathcal{I}_{A^{\prime}}$ is guaranteed to exist. 
then

$$
\operatorname{VIEW}_{B^{\prime}}^{A, B^{\prime}}\left(x_{0}, \cdot, y, r_{B}\right)=\operatorname{VIEW}_{B^{\prime}}^{A, B^{\prime}}\left(x_{1}, \cdot, y, r_{B}\right) .
$$

Bob's Privacy: For every strategy $A^{\prime}, \forall x \in S_{1}, \forall y_{0}, y_{1} \in S_{2}, \forall r_{A}$,

$$
\mathrm{VIEW}_{A^{\prime}}^{A^{\prime}, B}\left(x, r_{A}, y_{0}, \cdot\right)=\operatorname{VIEW}_{A^{\prime}}^{A^{\prime}, B}\left(x, r_{A}, y_{1}, \cdot\right) \text {. }
$$

Note that security against malicious parties implies security against honestbut-curious parties. That is,

Fact 1 If a protocol securely computes the function $f$ in the unbounded malicious model, it securely computes $f$ in the unbounded honest-but-curious model.

Definition 3 (Trivial and non-trivial functions). A finite function $f$ is called trivial if there exists a protocol securely computing it in the unbounded malicious model; otherwise, $f$ is called non-trivial.

\subsection{The Combinatorial Characterization}

We prove that the trivial functions are exactly those that do not contain an insecure minor (a simple generalization of an embedded or CK89 14 ).

Definition 4 (Insecure minor). A function $f: S_{1} \times S_{2} \rightarrow S_{3}$ contains an insecure minor if there exist $x_{0}, x_{1} \in S_{1}, y_{0}, y_{1} \in S_{2}$, and $a, b, c \in S_{3}$ such that $b \neq c$, and $f\left(x_{0}, y_{0}\right)=f\left(x_{1}, y_{0}\right)=a, f\left(x_{0}, y_{1}\right)=b$, and $f\left(x_{1}, y_{1}\right)=c$. Graphically 15

$$
\begin{array}{|c|c|c|}
\hline \mathbf{f}: & \mathbf{x}_{\mathbf{0}} & \mathbf{x}_{\mathbf{1}} \\
\hline \mathbf{y}_{\mathbf{0}} & a & a \\
\hline \mathbf{y}_{\mathbf{1}} & b & c \\
\hline
\end{array}
$$

Examples. As immediately apparent from their tables, each of the AND and OT functions contain an insecure minor (and actually an embedded or):

\begin{tabular}{|c|c|c|}
\hline AND : & $\mathbf{0}$ & $\mathbf{1}$ \\
\hline $\mathbf{0}$ & 0 & 0 \\
\hline $\mathbf{1}$ & 0 & 1 \\
\hline
\end{tabular}

\begin{tabular}{|c|c|c|}
\hline $\mathbf{O T}:$ & $(\mathbf{0}, \mathbf{0})$ & $(\mathbf{0}, \mathbf{1})$ \\
\hline $\mathbf{0}$ & 0 & 0 \\
\hline $\mathbf{1}$ & 0 & 1 \\
\hline
\end{tabular}

Theorem 1. A function $f(\cdot, \cdot)$ is trivial if and only if $f$ does not contain an insecure minor.

14 An embedded or is an insecure minor in which $a=b$. As shown in CK89, having an embedded or implies non-triviality in the two-sided honest-but-curious model, and characterizes the Boolean non-trivial functions in this model.

15 This graphical convention will be used in the rest of the paper, namely a table where columns correspond to possible inputs for Alice, rows correspond to possible inputs for Bob, and the entries are the corresponding output values. 
Proof. We break the proof into two parts; Theorem 1 follows from the following Claim 11 and Claim 2

First, we assume that $f$ does not contain an insecure minor and prove that $f$ is trivial by constructing a protocol $(A, B)$ that securely computes $f$ against malicious unbounded parties. Fix any $x_{0} \in S_{1}$ and $y_{0} \in S_{2}$. The protocol $(A, B)$, described in Fig. Il has a single round of communication (one message sent from $A$ to $B$ ), and is deterministic (namely $A$ and $B$ ignore their random inputs).

Claim 1. If $f$ does not contain an insecure minor then it is trivial.

Proof. We prove our claim by showing that $f$ is securely computed against unbounded malicious parties by the following protocol $(A, B)$ described in Fig. $\amalg$

\section{Protocol $(A, B)$}

$A$, on input $x \in S_{1}$ :

send to Bob the message $a \stackrel{\text { def }}{=} f\left(x, y_{0}\right)$.

$B$, on input $y \in S_{2}$, upon receipt of the message $a$ from Alice:

Find the lexicographically first $x_{1}$ such that $f\left(x_{1}, y_{0}\right)=a$

Set the output $\operatorname{OUT}_{B}\left(y, r_{B}, a\right)$ to $f\left(x_{1}, y\right)$.

If no such $x_{1}$ exists, set the output $\operatorname{OUT}_{B}\left(y, r_{B}, a\right)$ to $f\left(x_{0}, y\right)$.

Fig. 1. A secure protocol (against unbounded malicious parties) for a function $f$ not containing an insecure minor.

We first prove the correctness of the protocol. Recall that $x$ and $y$ are the inputs held by honest Alice and honest Bob respectively. Correctness when both parties are honest follows since for any message $a=f\left(x, y_{0}\right)$ sent by honest Alice, an honest Bob finds $x_{1}$ such that $f\left(x, y_{0}\right)=f\left(x_{1}, y_{0}\right)$. Since $f$ does not contain an insecure minor then it must hold that $f(x, y)=f\left(x_{1}, y\right)$ (otherwise $x, x_{1}, y_{0}, y$ constitute an insecure minor). Thus, Bob's output - $f\left(x_{1}, y\right)$ - is correct.

To prove correctness when only Bob is honest, we first define the following input function $\mathcal{I}_{A^{\prime}}:\{0,1\}^{*} \rightarrow S_{3}$ where if there is no $x_{1}$ such that $a=f\left(x_{1}, y_{0}\right)$ then $\mathcal{I}_{A^{\prime}}\left(x, r_{A}, a\right)=x_{0}$ and otherwise $\mathcal{I}_{A^{\prime}}\left(x, r_{A}, a\right)$ is the lexicographically first $x_{1}$ such that $a=f\left(x_{1}, y_{0}\right)$. Notice that the input function $\mathcal{I}_{A^{\prime}}$ is the same for every adversary $A^{\prime}$. By the definition of $\mathcal{I}_{A^{\prime}}$ it always holds that $\operatorname{OUT}_{B}\left(y, r_{B}, a\right)=$ $f\left(\mathcal{I}_{A^{\prime}}\left(x, r_{A}, a\right), y\right)$ and correctness follows.

Alice's privacy follows by observing that all information sent to Bob, namely $f\left(x, y_{0}\right)$, can be computed by Bob alone from the output of the function $f(x, y)$. This is because Bob can find some $x^{\prime}$ such that $f\left(x^{\prime}, y\right)=f(x, y)$, and conclude that $f\left(x, y_{0}\right)=f\left(x^{\prime}, y_{0}\right)$ (since $f$ does not contain an insecure minor). Bob's privacy follows immediately from the fact that this is a one-round protocol, where Bob sends no information to Alice, and thus her view is clearly identical for any input he may have.

Let us now prove the second part of Theorem II

Claim 2. If a function $f$ is trivial then it does not contain an insecure minor. 
Proof. If $f$ is trivial, then there is a protocol $(A, B)$ securely computing $f$ against unbounded parties. In particular, by Fact Il Protocol $(A, B)$ securely computes $f$ against honest-but-curious unbounded parties. We assume, for sake of contradiction, that $f$ contains an insecure minor.

Let $x_{0}, x_{1}, y_{0}, y_{1}$ constitute an insecure minor of $f$, that is there are $a, b, c \in S_{3}$ such that $b \neq c$ and

$$
\begin{array}{|c|c|c|}
\hline \mathbf{f}: & \mathbf{x}_{\mathbf{0}} & \mathbf{x}_{\mathbf{1}} \\
\hline \mathbf{y}_{\mathbf{0}} & a & a \\
\hline \mathbf{y}_{\mathbf{1}} & b & c \\
\hline
\end{array}
$$

By Bob's privacy from Definition II

$$
\operatorname{VIEW}_{A}\left(x_{0}, r_{A}, y_{1}, \cdot\right)=\operatorname{VIEW}_{A}\left(x_{0}, r_{A}, y_{0}, \cdot\right)
$$

for every $r_{A}$. By ranging over all possible $r_{A}$ we get

$$
\operatorname{TRANS}\left(x_{0}, \cdot, y_{1}, \cdot\right)=\operatorname{TRANS}\left(x_{0}, \cdot, y_{0}, \cdot\right) \text {. }
$$

On the other hand, by Alice's privacy, since $f\left(x_{0}, y_{0}\right)=f\left(x_{1}, y_{0}\right)=a$,

$$
\operatorname{VIEW}_{B}\left(x_{0}, \cdot, y_{0}, r_{B}\right)=\operatorname{VIEW}_{B}\left(x_{1}, \cdot, y_{0}, r_{B}\right)
$$

for every $r_{B}$. Again, by ranging over all possible $r_{B}$ we get

$$
\operatorname{TRANS}\left(x_{0}, \cdot, y_{0}, \cdot\right)=\operatorname{TRANS}\left(x_{1}, \cdot, y_{0}, \cdot\right) .
$$

Finally, again by Bob's privacy

$$
\operatorname{TRANS}\left(x_{1}, \cdot, y_{0}, \cdot\right)=\operatorname{TRANS}\left(x_{1}, \cdot, y_{1}, \cdot\right)
$$

Thus, by transitivity,

$$
\operatorname{TRANS}\left(x_{0}, \cdot, y_{1}, \cdot\right)=\operatorname{TRANS}\left(x_{1}, \cdot, y_{1}, \cdot\right) .
$$

We next use the following proposition, proved by CK84 to hold for every protocol, to argue that this transcript is equally distributed even if we fix the random input of Bob.

Proposition 1. Let $u_{0}, u_{1}, v_{0}, v_{1}, r_{A, 0}, r_{A, 1}, r_{B, 0}, r_{B, 1}$ be inputs and random inputs such that

$$
\text { Then, } \quad \operatorname{TRANS}\left(u_{0}, r_{A, 0}, v_{1}, r_{B, 1}\right)=\operatorname{TRANS}\left(u_{1}, r_{A, 1}, v_{0}, r_{B, 0}\right)=t \text {. }
$$

In other words, if changing the inputs for both Alice and Bob yields the same transcript, then changing the input for Alice only (or Bob only) will also yield the same transcript.

Fix arbitrary random inputs $q_{A}$ and $q_{B}$, and let $t=\operatorname{TRANS}\left(x_{0}, q_{A}, y_{1}, q_{B}\right)$. By Equation II , there exist $q_{A}^{\prime}, q_{B}^{\prime}$ such that $\operatorname{TRANS}\left(x_{1}, q_{A}^{\prime}, y_{1}, q_{B}^{\prime}\right)=t$, which by Proposition II implies that $t=\operatorname{TRANS}\left(x_{1}, q_{A}^{\prime}, y_{1}, q_{B}\right)$. 
Now, by Proposition $\mathbb{I}$ it holds that

$$
\begin{aligned}
\operatorname{Pr}_{r_{A}, r_{B}} & {\left[\operatorname{TRANS}\left(x_{0}, \cdot, y_{1}, \cdot\right)=t\right] } \\
= & \underset{r_{A}}{\operatorname{Pr}}\left[\operatorname{TRANS}\left(x_{0}, \cdot, y_{1}, q_{B}\right)=t\right] \cdot \underset{r_{B}}{\operatorname{Pr}}\left[\operatorname{TRANS}\left(x_{0}, q_{A}, y_{1}, \cdot\right)=t\right],
\end{aligned}
$$

and similarly

$$
\begin{aligned}
\operatorname{Pr}_{A}, r_{B} & {\left[\operatorname{TRANS}\left(x_{1}, \cdot, y_{1}, \cdot\right)=t\right] } \\
\quad= & \underset{r_{A}}{\operatorname{Pr}}\left[\operatorname{TRANS}\left(x_{1}, \cdot, y_{1}, q_{B}\right)=t\right] \cdot \underset{r_{B}}{\operatorname{Pr}}\left[\operatorname{TRANS}\left(x_{1}, q_{A}^{\prime}, y_{1}, \cdot\right)=t\right] .
\end{aligned}
$$

By Proposition II for every $r_{B}$

$$
\operatorname{TRANS}\left(x_{0}, q_{A}, y_{1}, r_{B}\right)=t \text { if and only if } \operatorname{TRANS}\left(x_{1}, q_{A}^{\prime}, y_{1}, r_{B}\right)=t
$$

Hence,

$$
\operatorname{Pr}_{r_{B}}\left[\operatorname{TRANS}\left(x_{0}, q_{A}, y_{1}, \cdot\right)=t\right]=\operatorname{Pr}_{r_{B}}\left[\operatorname{TRANS}\left(x_{1}, q_{A}^{\prime}, y_{1}, \cdot\right)=t\right] .
$$

Since by II $\operatorname{Pr}_{r_{A}, r_{B}}\left[\operatorname{TRANS}\left(x_{0}, \cdot, y_{1}, \cdot\right)=t\right]=\operatorname{Pr}_{r_{A}, r_{B}}\left[\operatorname{TRANS}\left(x_{1}, \cdot, y_{1}, \cdot\right)\right.$ $=t]$, and from 2, 3, 4 , we get that, for every $q_{B}$ and $t$,

$$
\underset{r_{A}}{\operatorname{Pr}}\left[\operatorname{TRANS}\left(x_{0}, \cdot, y_{1}, q_{B}\right)=t\right]=\operatorname{Pr}_{r_{A}}\left[\operatorname{TRANS}\left(x_{1}, \cdot, y_{1}, q_{B}\right)=t\right] .
$$

That is,

$$
\operatorname{TRANS}\left(x_{0}, \cdot, y_{1}, q_{B}\right)=\operatorname{TRANS}\left(x_{1}, \cdot, y_{1}, q_{B}\right)
$$

for every $q_{B}$.

Recall that the view of Bob is defined as his input, his random input, and the transcript of the communication. By Equation 5, for every $q_{B}$ the communication transcript between Alice and Bob is identically distributed when the inputs of Alice and Bob are $x_{0}, y_{1}$ and when their inputs are $x_{1}, y_{1}$. In both cases Bob has the same input $y_{1}$ and random input $q_{B}$, so Bob's view is identically distributed in both cases, namely for every $q_{B}$ it holds that

$$
\operatorname{VIEW}_{B}\left(x_{0}, \cdot, y_{1}, q_{B}\right)=\operatorname{VIEW}_{B}\left(x_{1}, \cdot, y_{1}, q_{B}\right) .
$$

Equation 6 contradicts the correctness requirement from Definition 11 because $f\left(x_{0}, y_{1}\right)=b \neq c=f\left(x_{1}, y_{1}\right)$, whereas the identical distributions of Bob's view imply that Bob has the same output distribution in both cases. Thus, we have reached a contradiction, which concludes the proof of the claim.

Claim II and Claim $\boldsymbol{\nu}$ complete the proof of Theorem II

\subsection{The Round Complexity of Secure Computation against Unbounded Malicious Parties}

Typically, multiple rounds and probabilism are crucial ingredients of secure computation. As stated in the following corollary, however, two-party secure computation in the unbounded malicious model is an exception. 
Corollary 1. If a function $f$ is securely computable in the unbounded malicious model, then it is so computable by a deterministic single-round (actually, singlemessage) protocol.

Proof. The corollary follows immediately from our proof of Theorem II (rather than from its statement). That proof, shows that, if a function $f$ is computable in the unbounded two-party malicious model, then it is so computed by the protocol of Fig. 11 in which only a single message is exchanged (from $A$ to $B$ ).

Together with the above corollary, our proof of Theorem II (actually, of Claim 2 alone) also immediately implies the following relationship between secure computation in the unbounded honest-but-curious model and in the unbounded malicious one.

Corollary 2. For every two-argument function $f$, one of the following holds: Either

1. $f$ is securely computable deterministically and in one round in the unbounded malicious model; Or

2. $f$ is not securely computable in the unbounded honest-but-curious model, even by probabilistic and multi-round protocols.

\section{Characterization of Complete Functions}

In this section we prove that every function that contains an insecure minor is complete for secure computation. That is, every non-trivial function is complete.

We shall consider secure computation in the (computationally) bounded malicious model. That is, the computation is secure provided that the (malicious) parties run in polynomial time. Thus, for the privacy conditions to hold, the appropriate probability distributions are only required to be indistinguishable by polynomial time Turing Machines (rather than identical as in the unbounded case of Definition 2 2 . In our proof we also consider the bounded honest-butcurious model. For lack of space we do not give precise definitions of secure computation in the bounded models, definitions which are much more involved and complex than the definitions in the unbounded models. Such definitions can be found, e.g., in Gol98. We note that our results hold for all reasonable definitions of secure computation in these model.

\subsection{Reductions and Completeness}

As usual, the definition of completeness relies on that of a reduction.

Definition 5 (Reductions). Let $f(\cdot, \cdot)$ and $g(\cdot, \cdot)$ be finite functions. We say that the function $g$ reduces to $f$ in the bounded malicious model (respectively, in the bounded honest-but-curious model) if there exists a transformation $1 \mathrm{f}$ from

\footnotetext{
${ }^{16}$ All reductions presented in this paper consist of efficient and uniform transformations.
} 
any protocol securely computing $f$ in the bounded malicious model (respectively, in the bounded honest-but-curious model) to a protocol securely computing $g$ in the same model.

Definition 6 (Completeness). The function $f(\cdot, \cdot)$ is complete for bounded malicious secure computations (respectively, bounded honest-but-curious secure computations) if every finite function $g(\cdot, \cdot)$ reduces to $f$ in the bounded malicious model (respectively, in the bounded honest-but-curious model).

Informally, a function $g$ reduces to a function $f$ if a secure protocol for $f$ can be converted to a secure protocol for $g$ without any additional assumptions. (Even more informally, $f$ is "harder" to compute securely than $g$.)

\subsection{Our Reduction vs. Black-Box Reductions}

As mentioned in the introduction, our reductions are not black-box ones, but are natural and very suitable for investigating which assumptions are sufficient for secure computation. In contrast, black-box reductions are not strong enough to establish our main theorem, the all-or-nothing nature of two-party secure computation. For instance, the (non-trivial) OR function is not complete under black-box reductions Kil99 17. Hence, black-box reductions do not give any indication regarding which is the minimal assumption necessary for implementing OR securely. On the other hand, using our notions of reductions and completeness, our main theorem implies that the complexity assumptions necessary for implementing OR securely are exactly the same as for all other non-trivial functions.

Let us now emphasize that our reductions and completeness satisfy basic expected properties of such notions.

Lemma 1. Let $f$ and $g$ be finite functions such that $g$ reduces to $f$ in the bounded malicious model of secure computation. Then any assumption sufficient for computing $f$ securely in the bounded malicious model is also sufficient for securely computing $g$ in the same model.

Proof. Consider a protocol $\left(A_{f}, B_{f}\right)$ that securely computes $f$ under some assumption $\mathrm{ASSUM}_{f}$. Since $g$ reduces to $f$, we can apply the transformation from $\left(A_{f}, B_{f}\right)$ to obtain a protocol $\left(A_{g}, B_{g}\right)$ such that if $\left(A_{f}, B_{f}\right)$ securely computes $f$ then $\left(A_{g}, B_{g}\right)$ securely computes $g$. Thus, if $\mathrm{ASSUM}_{f}$ holds then $\left(A_{g}, B_{g}\right)$ securely computes $g$.

Furthermore, by definition, these reductions are transitive:

$\overline{17}$ Indeed, it is not hard to see that in any protocol that uses a black-box for OR, the party receiving the output can input 0 to the black-box, thus obtaining the other party's input, without any way of being detected. Since this cannot be prevented, any protocol which is unconditionally secure using an OR black-box can be transformed into an unconditionally secure protocol, implying that only trivial functions can be black-box reduced to OR. 
Lemma 2. Let $f, g$, and $h$ be finite functions. If $h$ reduces to $g$ and $g$ reduces to $f$ then $h$ reduces to $f$.

Lemma 3. Let $f$ and $g$ be any finite functions. If $g$ can be computed securely in the bounded malicious model without any assumptions then $g$ reduces to $f$ in the bounded malicious model.

Proof. Consider a protocol $\left(A_{g}, B_{g}\right)$ that computes $g$ securely. The transformation from any protocol $\left(A_{f}, B_{f}\right)$ securely computing $f$ to a protocol securely computing $g$ ignores $\left(A_{f}, B_{f}\right)$ and outputs $\left(A_{g}, B_{g}\right)$.

Remark 1. We stress that our notion of completeness highlights the all-or-nothing nature of secure computation. Furthermore, by Yaose GMW 87, if factoring is hard or if trapdoor permutations exist, then all finite functions (even the trivial ones!) are complete.

\subsection{Main Theorem}

Theorem 2. If $f(\cdot, \cdot)$ is a non-trivial function, then $f$ is complete in the bounded malicious model.

Proof Outline. Although we aim towards the bounded malicious model, our proof of Theorem $\boldsymbol{\nu}$ wanders through the bounded honest-but-curious model (more direct proofs seem problemati 18). We first prove that every non-trivial function is complete in the honest-but-curious model. We then use standard techniques of GMW87 to transform any secure protocol in the bounded honestbut-curious model into a secure protocol in the bounded malicious model. In general this transformation requires some complexity assumptions, however in our case the protocol in the honest-but-curious model implies these assumptions. Thus, combining the above steps, every non-trivial function is complete in the malicious model.

Proof. We start by proving the analogue of Theorem 2 for the honest-but-curious model.

Claim 3. If a function $f(\cdot, \cdot)$ contains an insecure minor, then $f$ is complete in the bounded honest-but-curious model.

Proof. It is proven in GV87 that OT is complete in the bounded honest-butcurious model. Therefore, to establish our claim it suffices to prove that whenever $f$ contains an insecure minor then OT reduces to $f$.

Let $\left(A_{f}, B_{f}\right)$ be a secure protocol computing the function $f$ in the bounded honest-but-curious model. Because the function $f$ contains an insecure minor, there are values $x_{0}, x_{1}, y_{0}, y_{1}, a, b$ and $c$ such that $b \neq c, f\left(x_{0}, y_{0}\right)=f\left(x_{1}, y_{0}\right)=a$, $f\left(x_{0}, y_{1}\right)=b$, and $f\left(x_{1}, y_{1}\right)=c$. 


\section{Protocol $\left(A_{\mathrm{OT}}, B_{\mathrm{OT}}\right)$}

$A_{\mathrm{OT}}$ 's input: $\beta_{0}, \beta_{1} \in\{0,1\}$

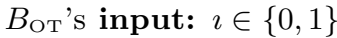

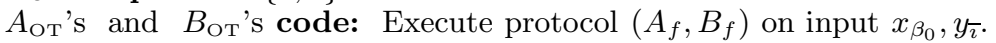

Denote by $z_{0}$ the output of $B_{f}$.

Execute protocol $\left(A_{f}, B_{f}\right)$ on input $x_{\beta_{1}}, y_{\imath}$.

Denote by $z_{1}$ the output of $B_{f}$.

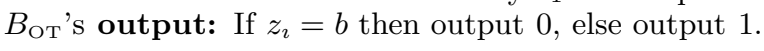

Fig. 2. A secure protocol in the bounded honest-but-curious model for computing OT from a function $f$ containing an insecure minor with values $x_{0}, x_{1}, y_{0}, y_{1}, a, b, c$.

In Fig. $\boldsymbol{Z}$ we describe a protocol $\left(A_{\mathrm{OT}}, B_{\mathrm{OT}}\right)$ which securely computes OT using this insecure minor and the protocol $\left(A_{f}, B_{f}\right)$.

In Protocol $\left(A_{\mathrm{OT}}, B_{\mathrm{OT}}\right)$ it holds that $z_{\imath}=f\left(x_{\beta_{\imath}}, y_{1}\right)$, and, thus, $z_{\imath}=b$ if $\beta_{\imath}=0$ (and $z_{\imath}=c \neq b$ otherwise), implying that the output of $B_{\mathrm{OT}}$ is correct. We next argue that the privacy constrains are satisfied for bounded honest-butcurious parties $A_{\text {От }}$ and $B_{\text {От }}$. First note that the only messages exchanged in $\left(A_{\mathrm{OT}}, B_{\mathrm{OT}}\right)$ are during the executions of $\left(A_{f}, B_{f}\right)$. Since $\left(A_{f}, B_{f}\right)$ computes $f$ securely, $A_{f}$ (and thus $A_{\text {от }}$ ) does not learn any information about $\imath$. Recall that $B_{f}$ is not allowed to learn any information that is not implied by his input and the output of the function. In the case of OT, this means $B_{\text {От }}$ should not learn any information about $\beta_{\bar{\imath}}$. However, the only information that $A_{\mathrm{OT}}$ sends that depends on $\beta_{\bar{\imath}}$ are during the execution of $\left(A_{f}, B_{f}\right)$ on input $\left(x_{\beta_{\bar{\tau}}}, y_{0}\right)$ and, thus, $z_{\bar{\imath}}=a$ for both values of $\beta_{\bar{\imath}}$. By the fact that $\left(A_{f}, B_{f}\right)$ computes $f$ securely, $B_{f}$ does not learn any information on $\beta_{\bar{\imath}}$.

Note that the above protocol is secure only if $B_{\text {Oт }}$ is honest. Also note that in protocol $\left(A_{\mathrm{OT}}, B_{\text {От }}\right)$ it is important that only $B_{f}$ gets the outputs $z_{0}$ and $z_{1}$ of $\left(A_{f}, B_{f}\right)$. That is, if $A_{\text {От }}$ gets $z_{0}$ or $z_{1}$ then she can learn $B_{\text {От 's input for at }}$ least one of the possible values of her input (since either $b \neq a$ or $c \neq a$ or both).

Let us now prove an "hybrid result" bridging the completeness in the two bounded models of secure computation.

Claim 4. Let $f(\cdot, \cdot)$ be any finite function. If $f$ is complete in the bounded honestbut-curious model then it is complete in the bounded malicious model.

Proof Sketch. Let $g$ be any finite function. We need to prove that $g$ reduces to $f$ in the bounded malicious model. We are promised that $g$ reduces to $f$ in the bounded honest-but-curious model. That is, there is a transformation from any protocol securely computing $f$ to one securely computing $g$ in the bounded honest-but-curious model.

To obtain a protocol securely computing $g$ in the malicious model, we proceed as follows. First, there exists a transformation mapping any protocol that

$\overline{18}$ For example, we cannot use Kilian's reduction Kil.91 from OT to a two-sided computation of $\mathrm{OR}$ in the bounded malicious model. 
securely computes OT in the bounded honest-but-curious model into a one-way function IL 89 . Second, since $f$ is complete in the bounded honest-but-curious model, this implies that there exists a transformation mapping any protocol that securely computes $f$ in the bounded honest-but-curious model into a one-way function. Third, one-way functions imply pseudo-random generators HILLYI, which in turn imply bit commitment Na.089. Finally, bit commitment implies that it is possible to transform any protocol securely computing an arbitrary function $g$ in the bounded honest-but-curious model into a protocol securely computing $g$ in the bounded malicious model GMW87. Putting the above together, we obtain a transformation from a protocol securely computing $f$ in the bounded honest-but-curious model to one computing the function $g$ in the bounded malicious model.

We are ready to complete the proof of Theorem $\boldsymbol{\nabla}$ By Theorem II any nontrivial function $f$ contains an insecure minor. Thus, by Claim 3 and Claim $\square f$ is complete in the bounded malicious model.

\section{Acknowledgments}

We thank Joe Kilian and Eyal Kushilevitz for insightful comments. We also thank Lior Pachter for risking his life to save a preliminary version of this work.

\section{References}

Blu82. M. Blum. Coin flipping by phone. IEEE Spring COMPCOM, pages 133$137,1982$.

BGW88. M. Ben-Or, S. Goldwasser, and A. Wigderson. Completeness theorems for noncryptographic fault-tolerant distributed computations. In Proc. of the 20th Symp. on Theory of Computing, pages 1-10, 1988.

CCD88. D. Chaum, C. Crépeau, and I. Damgard. Multiparty unconditionally secure protocols. In Proc. of the 20th Symp. on Theory of Comp., pages 11-19, 1988 .

CK88. C. Crépeau and J. Kilian. Achieving oblivious transfer using weakened security assumptions. In Proc. of the 29th IEEE Symp. on Foundations of Computer Science, pages 42-52, 1988.

CK89. B. Chor and E. Kushilevitz. A zero-one law for Boolean privacy. SIAM J. on Discrete Math., 4(1):36-47, 1991. Prelim. version in STOC '89, 1989.

Cré88. C. Crépeau. Equivalence between two flavors of oblivious transfers. In Advances in Cryptology - CRYPTO '87, volume 293 of Lecture Notes in Computer Science, pages 350-354. Springer, 1988.

EGL85. S. Even, O. Goldreich, and A. Lempel. A randomized protocol for signing contracts. CACM, 28(6):637-647, 1985.

FMR84. M. J. Fischer, S. Micali, and C. Rackoff. A secure protocol for the oblivious transfer. Presented in EUROCRYPT '84, 1984. Printed version in $J$. of Cryptology, 9(3):191-195, 1996.

GMR85. S. Goldwasser, M. Micali, and C. Rackoff. The knowledge complexity of interactive proof systems. SIAM J. on Computing, 18:186-208, 1989. Preliminary version in STOC '85, 1985.

GMW86. O. Goldreich, S. Micali, and A. Wigderson. Proofs that yield nothing but their validity, or all languages in NP have zero-knowledge proof systems. In J. ACM, 38(1):691-729, 1991. Preliminary version in FOCS '86, 1986. 
GMW87. O. Goldreich, S. Micali, and A. Wigderson. How to play any mental game. In Proc. of the 19th Symp. on the Theory of Comp., pages 218-229, 1987.

Gol98. O. Goldreich. Secure multi-party computation (working draft). Available from http://www.wisdom.weizmann.ac.il/ oded/foc.html, 1998.

GV87. O. Goldreich and R. Vainish. How to solve any protocol problem - an efficiency improvement. In Advances in Cryptology - CRYPTO '87, volume 293 of Lecture Notes in Computer Science, pages 73-86. Springer, 1988.

HILL91. J. Hastad, R. Impagliazzo, L. A. Levin, and M. Luby. Construction of a pseudo-random generator from any one-way function. Technical Report TR-91-068, International Computer Science Institute. 1991.

IL89. R. Impagliazzo and M. Luby. One-way functions are essential for complexity based cryptography. In Proc. of the 30th IEEE Symp. on Foundations of Computer Science, pages 230-235, 1989.

IR89. R. Impagliazzo and S. Rudich. Limits on the provable consequences of one-way permutations. In Proc. of the 21st ACM Symp. on the Theory of Computing, pages 44-61, 1989.

Kil88. J. Kilian. Basing cryptography on oblivious transfer. In Proc. of the 20th ACM Symp. on the Theory of Computing, pages 20-31, 1988.

Kil90. J. Kilian. Uses of Randomness in Algorithms and Protocols. MIT Press, 1990.

Kil91. J. Kilian. A general completeness theorem for two-party games. In Proc. of the 23th ACM Symp. on the Theory of Computing, pages 553-560, 1991.

Kil99. J. Kilian. Personal communication. 1999.

KKMO98. J. Kilian, E. Kushilevitz, S. Micali, and R. Ostrovsky. Reducibility and completeness in private computations. 1998. To appear in SIAM J. on Computing. This is the journal version of Kil91 KMO94.

KMO94. E. Kushilevitz, S. Micali, and R. Ostrovsky. Reducibility and completeness in multi-party private computations. In Proc. of the 35th IEEE Symp. on Foundations of Computer Science, pages 478-491, 1994.

Kus89. E. Kushilevitz. Privacy and communication complexity. SIAM J. on Discrete Mathematics, 5(2):273-284, 1992. Preliminary version in FOCS '89, 1989.

MR92. S. Micali and P. Rogaway. Secure computation. In Advances in Cryptology - CRYPTO '91, vol. 576 of Lecture Notes in Computer Science, pages 392404. Springer-Verlag, 1992. An updated version presented at: Workshop on Multi-Party Secure Computation, Weizmann Inst., Israel, June 1998.

Nao89. M. Naor. Bit commitment using pseudorandom generators. J. of Cryptology, 4:151-158, 1991. Preliminary version in Advances in CryptologyCRYPTO '89, 1989.

Rab81. M. O. Rabin. How to exchange secrets by oblivious transfer. Technical Report TR-81, Harvard Aiken Computation Laboratory, 1981.

Yao82. A. C. Yao. Protocols for secure computations. In Proc. of the 23th IEEE Symp. on Foundations of Computer Science, pages 160-164, 1982.

Yao86. A. C. Yao. How to generate and exchange secrets. In Proc. of the 27th IEEE Symp. on Foundations of Computer Science, pages 162-167, 1986. 